\title{
Cosmological Issues for Revised Canonical Quantum Gravity
}

\author{
Giovanni Montani \\ ICRA - International Center for Relativistic Astrophysics \\ Dipartimento di Fisica (G9), \\ Università di Roma, "La Sapienza", \\ Piazzale Aldo Moro 5, 00185 Rome, Italy. \\ e-mail: montani@icra.it
}

PACS 83C

\begin{abstract}
In a recent work [1] we presented a reformulation of the canonical quantum gravity, based on adding the so-called kinematical term to the gravity-matter action; this revised approach leads to a self-consistent canonical quantization of the 3-geometries, referred to the external time as provided via the added term.

Here, we show how the kinematical term can be interpreted in terms of a non relativistic dust fluid which plies the role of a "real clock' for the quantum gravity theory, and, in the WKB limit of a cosmological problem, makes account for a dark matter component which, at present time, could play a dynamical role.
\end{abstract}




\section{Fundamental Remarks}

The Canonical Method of quantization relies on the existence of an Hamiltonian function for the system, regarded as conjugate variable to the physical time, and is implemented by recognizing the dynamics admits an Hamiltonian constraint; in fact, the quantum dynamics is easily obtained by the transcription of such a constraint via the operators associated to the canonical variables [2].

As an helpful example for the analysis below developed, we review the case of the one-dimensional non-relativistic (parametrized) particle, whose action reads

$$
S=\int\{p \dot{q}-h(p, q)\} d t
$$

where $t$ denotes the Newton time and $h$ the Hamiltonian function. In order to quantize this system, we parameterize the Newton time as $t=t(\tau)$, so getting the new action as

$$
S=\int\left\{p \frac{d q}{d \tau}-h(p, q) \frac{d t}{d \tau}\right\} d \tau .
$$

Now we set $p_{0} \equiv-h$ and add this relation to the above action by a Lagrangian multiplier $\lambda$, i.e.

$$
S=\int\left\{p \frac{d q}{d \tau}+p_{0} \frac{d t}{d \tau}-\bar{h}\left(p, q, p_{0}, \lambda\right)\right\} d \tau \quad \bar{h} \equiv \lambda\left(h+p_{0}\right) .
$$

By varying this action with respect to $p$ and $q$, we get the Hamilton equations $d q / d \tau=$ $\lambda \partial h / \partial p$ and $d p / d \tau=-\lambda \partial h / \partial q$, while the variations of $p_{0}$ and $t$ yield $d t / d \tau=\lambda$ and $d p_{0} / d \tau=0$; all together, these equations describe the same Newton dynamics, having the energy as constant of the motion. But now, by varying $\lambda$, we get the (desired) constraint $h+p_{0}=0$, which, in terms of the operators $\hat{p}_{0}=-i \hbar \partial_{t}$ and $\hat{h}$, provides the Schrödinger equation $i \hbar \partial_{t} \psi=\hat{h} \psi$, as taken for the system state function $\psi(t, q)$. Finally we remark that, when retaining the relation $d t / d \tau=\lambda$, we are able to write the wave equation in the parametric time as

$$
i \hbar \partial_{\tau} \psi(\tau, q)=\lambda(\tau) \hat{h} \psi(\tau, q),
$$

where $\lambda(\tau)$ is to be assigned.

In spite of its simplicity, this example is a naive, but very good prototype of our approach to the canonical quantum gravity.

When considering the gravitational field, the notion of an Hamiltonian function is recognized, as soon as, the four-dimensional manifold $\mathcal{M}^{4}$ is splitted into a one-parameter family of spatial hypersurfaces $\Sigma^{3}$, i.e. $\mathcal{M}^{4}=\Sigma^{3} \otimes R[3]$.

Thus, if $\mathcal{M}^{4}$ admits generic internal coordinates $y^{\rho}$ and a metric tensor $g_{\mu \nu}\left(y^{\rho}\right)(\mu, \nu, \rho=$ $0,1,2,3)$, then we can chose a family of space-like hypersurfaces filling $\mathcal{M}^{4}$, by assigning the parametric equations $y^{\rho}=y^{\rho}\left(t, x^{i}\right)(i=1,2,3)$; so doing, we obtain a new basis, composed of the normal field $n^{\mu}\left(y^{\rho}\right)$ and the tangent vectors $e_{i}^{\mu} \equiv \partial_{i} y^{\mu}$ to $\Sigma^{3}\left(g_{\mu \nu} n^{\mu} n^{\nu}=\right.$ $\left.-1, g_{\mu \nu} n^{\mu} e_{i}^{\nu}=0\right)$, on which, we can project the deformation vector $N^{\mu} \equiv \partial_{t} y^{\mu}$ as $\partial_{t} y^{\mu}=N n^{\mu}+N^{i} \partial_{i} y^{\mu}$ (where $N$ and $N^{i}$ are respectively called the lapse function and 
the shift vector).

Now we may regard the parameterization $y^{\rho}=y^{\rho}\left(t, x^{i}\right)$, as a coordinate transformation between two coordinates systems and, observing that the 3 -metric induced on $\Sigma^{3}$ reads $h_{i j} \equiv g_{\mu \nu} \partial_{i} y^{\mu} \partial_{j} y^{\nu}$, finally, the line element admits the representation

$$
d s^{2}=g_{\mu \nu} d x^{\mu} d x^{\nu}=-N^{2} d t^{2}+h_{i j}\left(d x^{i}+N^{i} d t\right)\left(d x^{j}+N^{j} d t\right) .
$$

In the system of coordinates $t$ and $x^{i}$, the ten independent $g_{\mu \nu}$ are replaced by the ten independent functions $\left\{N, N^{i}, h_{i j}\right\}$, and the normal field takes the form $n^{\mu}=$ $\left\{1 / N,-N^{i} / N\right\}$.

When the Einstein-Hilbert and matter (below we consider a real scalar field) action is recasted in this system of coordinates, it happens that the variables $N$ and $N^{i}$, being cyclic ones, behave as Lagrange multipliers. As a consequence, we get eight constraints, corresponding to the vanishing of the conjugate momenta $p_{N}$ and $p_{N^{i}}$, as well as, of the super-Hamiltonian $\left(H^{g}+H^{\phi}\right)$ and of the super-momentum $\left(H_{i}^{g}+H_{i}^{\phi}\right)$ (the labels $g$ and $\phi$ refer respectively to the metric and the scalar field).

The canonical quantization of the system, is then performed by upgrading the 3-metric and its conjugate momentum to operators $\hat{h}_{i j}, \hat{\pi}^{i j}=-i \hbar \delta(\quad) / \delta h_{i j}$ (the same for the field variables leads to $\hat{\phi}$ and $\left.\hat{p}_{\phi}=-i \delta(\quad) / \delta \phi\right)$, and implementing the Hamiltonian constraint on a quantum level, i.e. $\left(\hat{H}^{g}+\hat{H}^{\phi}\right) \Psi=0 \quad\left(\hat{H}_{i}^{g}+\hat{H}_{i}^{\phi}\right) \Psi=0$. The former of these functional equations, known as the Wheeler-DeWitt one, provides the quantum dynamics, while the latter ensures the invariance under the 3-diffeomorphisms, i.e. the state function $\Psi$ depends on $\phi$ and a class of 3 -geometries $\left\{h_{i j}\right\}[4]^{1}$.

The most unsatisfactory features of the above formulation consist of the absence of a time evolution and the impossibility for an Hilbert space [2, 5] (see also [6] and [7, 8]); but it exists also a ground-level criticism: indeed, the above splitting procedure relies on the possibility to distinguish between space-like and time-like objects (for instance the normal field $n^{\mu}$ should be time-like), but, when $g_{\mu \nu}$ is a quantum field, these notions can be recognized at most in terms of "expectation values". Therefore, on a quantum level, the $(3+1)$-splitting makes sense only in a perturbative limit, when yet survives the concept of metric background. A different approach consists of determining the character of geometrical objects, before quantizing the system, i.e. fixing the reference frame, determining the space-like family of hypersurfaces $\Sigma^{3}$ and only then quantizing the dynamics.// The following three sections answer the fundamental questions concerning such a different point of view,i.e.: what physically it means? Which quantum dynamics it yields? Which cosmological issues it predicts?

\section{The Kinematical Action and the Reference Fluid}

To fix the reference frame, it is equivalent to assign the lapse function and the shift vector already in the action, and then varying only the 3-metric; but so doing, we loss

\footnotetext{
${ }^{1}$ The fact that, the wave functional is independent of $N$ and $N^{i}$, reflects the classical constraints $p_{N}=0$ add $p_{N^{i}}=0$.
} 
the super-Hamiltonian and the super-momentum constraints and, though, we have to do with $\infty^{3}$ degrees of freedom, nevertheless our situation becomes very similar to that one analyzed for a non-relativistic particle. Thus, in close analogy to such case, we parametrized the gravity-matter action by means of the so-called "kinematical term" [2], which refers the dynamics to the generic coordinates $y^{\rho}$, playing here the role that above was proper of the Newton time. The extended action reads [1]

$$
S^{g \phi k}=\int_{\mathcal{M}^{4}}\left\{\pi^{i j} \partial_{t} h_{i j}+\pi_{\phi} \partial_{t} \phi+p_{\mu} \partial_{t} y^{\mu}-N\left(H^{g}+H^{\phi}+p_{\mu} n^{\mu}\right)-N^{i}\left(H_{i}^{g}+H_{i}^{\phi}+p_{\mu} \partial_{i} y^{\mu}\right)\right\} d^{3} x d t .
$$

Above, the (normal) field $n^{\mu}\left(y^{\rho}\right)$ is, on this level, to be regarded as a generic one and therefore assigned arbitrarily; $\partial_{i} y^{\mu}$ is a potential-like term, $p_{\mu}$ is determined by the field equations and anyway all the added terms are metric independent.

By varying this action with respect to $h_{i j} \pi_{i j} \phi \pi_{\phi}$, we get the field equations as unchanged, while the variation of $N$ and $N^{i}$ provides the new (desired) constraints

$$
H^{g}+H^{\phi}=-p_{\mu} n^{\mu} \quad H_{i}^{g}+H_{i}^{\phi}=-p_{\mu} \partial_{i} y^{\mu}
$$

Finally, the variation of the kinematical variables $y^{\mu}$ and $p_{\mu}$, provides the additional equations

$$
\partial_{t} y^{\mu}=N n^{\mu}+N^{i} \partial_{i} y^{\mu} \quad \partial_{t} p_{\mu}=-N p_{\rho} \partial_{\mu} n^{\rho}+\partial_{i}\left(N^{i} p_{\mu}\right) .
$$

Since $n^{\mu}$ is assigned $a b$ initio, then the specification of $N$ and $N^{i}$ allows to solve the first of these equations for $y^{\mu}\left(t, x^{i}\right)$ and hence, the second one, yields the generalized momentum $p_{\mu}\left(t, x^{i}\right)$ (entering linearly in the Hamiltonian).

Now, to understand the physical meaning of the added kinematical term, we have to investigate these field equations by restoring their covariant form, via the variables $y^{\mu}$. To this end, we denote the coordinates $\left(t, x^{i}\right)$ by barred indices $\bar{\mu}, \bar{\nu}, \bar{\rho}, \ldots$ and we remark that the following relations take place: $\partial_{t}=\partial_{t} y^{\mu} \partial_{\mu} \partial_{i}=\partial_{i} y^{\mu} \partial_{\mu} n^{\bar{\mu}} \partial_{\bar{\mu}}=n^{\mu} \partial_{\mu}$. Then the first of equations (8) rewrites as $n^{\mu}=n^{\bar{\rho}} \partial_{\bar{\rho}} y^{\mu}$; this equation is crucial to ensure that, after the variation, $n^{\mu}$ is a real unit time-like vector, i.e.

$$
g_{\mu \nu} n^{\mu} n^{\nu}=g_{\mu \nu} n^{\bar{\rho}} \partial_{\bar{\rho}} y^{\mu} n^{\bar{\sigma}} \partial_{\bar{\sigma}} y^{\nu}=g_{\bar{\rho} \bar{\sigma}} n^{\bar{\rho}} n^{\bar{\sigma}}=-1,
$$

the last equality being true by construction of $g_{\bar{\mu} \bar{\nu}}$ and $n^{\bar{\mu}}$; in fact the metric form $g_{\bar{\mu} \bar{\nu}}$, as given in the line element (5) , ensures the normal vector $n^{\bar{\mu}} \equiv\left(1 / N,-N^{i} / N\right)$ be a unit timelike one.

Thus, after the variation of the action, our approach ensures, differently from the Wheeler-DeWitt one, that we have to do, even on a quantum level. with a real normal field, as far as the first kinematical equation holds.

Being $n^{\mu} p_{\mu}$ a 3 -scalar density of weight $1 / 2$, we may set $n^{\mu} p_{\mu} \equiv-\omega\left(t, x^{i}\right)$, and then define the real scalar $\varepsilon \equiv-\omega / \sqrt{h}$ (with $\left.h \equiv \operatorname{det} h_{i j}\right)$.

Using these information and the first of (18), we may rewrite the second one as 


$$
n^{\rho}\left[\partial_{\rho}\left(N p_{\mu}\right)-\partial_{\mu}\left(N p_{\rho}\right)\right]=-\partial_{\mu}(N \sqrt{h} \varepsilon)+p_{\mu}\left(n^{\rho} \partial_{\rho} N+\partial_{i} N^{i}\right) .
$$

Since the fundamental aim of our reformulation, apart from removing the ambiguity about the time-like character of the normal, consists of constructing a quantum gravity theory having evolution, there are no serious reasons to deform the super-momentum constraint; indeed, as we shall see better in the next section, the "frozen formalism" is removed as soon as the Super-Hamiltonian is no longer zero. Therefore we can require the condition $p_{\mu} \partial_{i} y^{\mu} \equiv p_{i}=0$ holds, so getting $p_{\mu}=\omega n_{\mu}=-\sqrt{h} \varepsilon n_{\mu}$; by other words, such a conservative choice, is equivalent to take the generalized momentum as parallel to the normal field, and therefore time-like. The above form of the generalized momentum is preserved by the dynamics (i.e., once it is assigned as a cauchy problem, then the second of the kinematical equations ensures it holds for ever), under the constraint $\partial_{i} N=0$. In fact, by multiplying the second of equations (8) by $\partial_{i} y^{\mu}$, we get, after some algebra, the following equation for $p_{i}$

$$
\partial_{t} p_{i}-p_{j} \partial_{i} N^{j}-\epsilon \sqrt{h} \partial_{i} N=0 .
$$

We see how the above condition transforms (11) into a linear and homogeneous partial differential equation (in normal form) in the unknowns $p_{i}$; if we set the initial condition $p_{i}^{0}=0$, then the unique solution is $p_{i} \equiv 0$, valid for any later time.

Remarking that, in the barred coordinates $N \sqrt{h}=\sqrt{-\bar{g}}$, the above restriction $p_{\mu}=$ $-\sqrt{h} \varepsilon n_{\mu}$ reduces equation (10), via some technical steps, to the form

$$
\varepsilon n^{\rho}\left(\partial_{\rho} n_{\mu}-\partial_{\mu} n_{\rho}\right)+\frac{n_{\mu}}{\sqrt{-g}} \partial_{\rho}\left(\sqrt{-g} \varepsilon n^{\rho}\right)=0 .
$$

The above equation reads covariantly as

$$
\varepsilon n^{\rho}\left(\nabla_{\rho} n_{\mu}-\nabla_{\mu} n_{\rho}\right)+n_{\mu} \nabla_{\rho}\left(\varepsilon n^{\rho}\right)=0
$$

which, since $n^{\rho} \nabla_{\mu} n_{\rho}=0$ (being $n^{\mu}$ a unit vector), finally becomes

$$
\varepsilon n^{\rho} \nabla_{\rho} n_{\mu}+n_{\mu} \nabla_{\rho}\left(\varepsilon n^{\rho}\right)=0=\nabla_{\rho} t_{\mu}^{\rho} .
$$

Thus we got the surprising result that, the kinematical momentum equation, reduces to the conservation law of a "dust energy-momentum tensor" $t_{\mu \nu}=\varepsilon n_{\mu} n_{\nu}$. Multiplying equation (14) by $n^{\mu}$, it implies the additional condition

$$
\nabla_{\rho}\left(\varepsilon n^{\rho}\right)=0
$$

this condition simplifies (14) to the form

$$
n^{\rho} \nabla_{\rho} n_{\mu}=0=n^{\rho}\left(\partial_{\rho} n_{\mu}-\frac{1}{2} n^{\sigma} \partial_{\mu} g_{\rho \sigma}\right) .
$$

As well known, the dynamical equations describing a perfect fluid characterized by energy density $\rho$, pressure $p$, entropy density $\sigma$ and four-velocity $u_{\mu}$, take the form 


$$
\begin{gathered}
\nabla_{\mu}\left(\sigma u^{\mu}\right)=0 \\
(\rho+p) u^{\rho}\left(\partial_{\rho} u_{\mu}-\frac{1}{2} u^{\sigma} \partial_{\mu} g_{\rho \sigma}\right)=-\partial_{\mu} p-u_{\mu} u^{\rho} \partial_{\rho} p .
\end{gathered}
$$

By comparing (15) with (17) and (16) with (18), we see how the "kinematical fluid" has $\rho \equiv \varepsilon$, zero pressure, entropy density proportional to the energy one (i.e. $\sigma \propto \varepsilon$ ) and four-velocity $n^{\mu}$; thus, on a classical level, our procedure is equivalent to introduce a real reference fluid behaving as a non-relativistic dust.

Since, being $n_{\mu} \partial_{i} y^{\mu}=0$, the energy-momentum tensor of such a dust is orthogonal to the hypersurfaces $\Sigma^{3}$, then it contributes only to the super-Hamiltonian, which rewrites as $H^{g}+H^{\phi}+\sqrt{h} \varepsilon$.

Above, we clarified the physical meaning of the kinematical action, so answering for the first posed question, in close analogy with the approach presented in [9] about the so-called "Gaussian reference fluid" (for a discussion on non-Gaussian fluid see [10, 11]). However, the notion of a reference fluid, here is preserved also in the (generic) coordinates system $\left\{t, x^{i}\right\}$, where, by assigning the functions $N$ and $N^{i}$, we get directly the normal field; in this case, equation (15) takes the simple form $\partial_{t} \omega+\partial_{i}\left(N^{i} \omega\right)=0$. In a synchronous (or Gaussian) reference, when the fluid is comoving with the coordinates $\left\{t, x^{i}\right\}\left(N=1 N^{i}=0 \Rightarrow n^{\bar{\rho}}=(1, \mathbf{0})\right)$, we get, as expected, $\omega=\omega\left(x^{i}\right) \Rightarrow \varepsilon=$ $-\omega\left(x^{i}\right) / \sqrt{h}$. It is worth noting how, the energy density of the reference fluid, has the opposite sign of the super-Hamiltonian and therefore is, in general, non-positive defined.

We conclude this section, devoted to the classical aspects of our reformulation, by stressing the following two points:

i) To fix the reference frame, i. e. the lapse function and the shift vector, via the kinematical action, gives rise to the appearance of a real dust fluid; by a suggestive language, we may claim that this "gauge fixing procedure" materializes the reference frame.

ii) All the dynamical information about the reference fluid, result to be contained in the second kinematical equation, while the first one seems to reflect simply a parameterization of the dynamics, so ensuring the self-consistency of the whole theory.

\section{Canonical Quantum Dynamics}

The quantum dynamics, corresponding to the action (6), is easily got by implementing the Hamiltonian constraint to their operator form, taken as acting on a wave functional $\Psi$, now depending even from $y^{\mu}$ (with $\hat{p}_{\mu}=-i \hbar \delta() / \delta y^{\mu}$ ). The classical restriction to take a generalized momentum parallel to the normal field, is translated on a quantum level, by preserving the super-momentum constraint in its form and writing down the field equations as (the general theory is nevertheless a viable issue)

$$
i \hbar n^{\mu} \frac{\delta \Psi}{\delta y^{\mu}}=\left(\hat{H}^{g}+\hat{H}_{i}^{\phi}\right) \Psi \quad\left(\hat{H}_{i}^{g}+\hat{H}_{i}^{\phi}\right) \Psi=0 \quad \Psi=\Psi\left(y^{\mu},\left\{h_{i j}\right\}, \phi\right) .
$$


where the wave functional is taken on the 3-geometries $\left\{h_{i j}\right\}$, i.e. a whole class of 3-metric tensors, connected via a 3-diffeomorphism.

In analogy to the case of the parametrized particle, we may rewrite this set of $\infty^{3}$ equations in the coordinate system $\left\{t, x^{i}\right\}$, as soon as, using the first kinematical equation; indeed, as above outlined, the use of this (classical) equation is justified, even on a quantum level, by observing that:

i) It is necessary to specify the meaning of the field $n^{\mu}$ in the above quantum dynamics; indeed, such a field becomes the real normal one and the system evolution is constrained to a fixed choice of $N$ and $N^{i}$.

ii) On a classical level, this equation plies no dynamical role and resembles very closely an $\infty^{3}$-version of the corresponding equation for the non-relativistic particle.

iii) Its use is justified a posteriori because it reproduces the expected Schrödinger equation.

So doing, we get

$$
i \hbar \partial_{t} y^{\mu} \frac{\delta \Psi}{\delta y^{\mu}}=N\left(\hat{H}^{g}+\hat{H}^{\phi}\right) \Psi
$$

By defining the operator $\partial_{t}() \equiv \int_{\Sigma^{3}} \partial_{t} y^{\mu} \delta() / \delta y^{\mu}$ we finally arrive to a single (smeared) Schrödinger equation

$$
i \hbar \partial_{t} \Psi=i \hbar \int_{\Sigma_{t}^{3}}\left\{\frac{\delta \Psi}{\delta y^{\mu}} \partial_{t} y^{\mu}\right\} d^{3} x=\hat{\mathcal{H}} \Psi \equiv\left[\int_{\Sigma_{t}^{3}} N\left(\hat{H}^{g}+\hat{H}^{\phi}\right) d^{3} x\right] \Psi
$$

with $\Psi=\Psi\left(t,\left\{h_{i j}\right\}, \phi\right)$. We regard this equation as the fundamental one of the revised canonical quantum gravity, which, once fixed $N$ and $N^{i}$ (the latter one does not enter in this equation), provides the dynamics of quantum 3-geometries and matter fields; the label time $t$ acquires a precise physical meaning in view of the analysis developed in the previous section, i.e. it is a real "fluid clock" filling the hypersurfaces $\Sigma_{t}^{3}$ (the label $t$ specifying each of them); however, to understand the way in which such a reference fluid manifests its presence on a quantum level, see the below discussion about the eigenvalues problem.

As shown in [1], by adopting a suitable normal ordering in the kinetic part of $\hat{H}^{g}$, i.e. $G_{i j k l} \pi^{i j} \pi^{k l} \rightarrow \delta / \delta h_{i j}\left(G_{i j k l} \delta / \delta h_{k l}\right)$, then we are able to turn the space of the solutions into an Hilbert one by the inner product $\left\langle\Psi_{1} \mid \Psi_{2}\right\rangle\left(\Psi_{1}\right.$ and $\Psi_{2}$ are generic functionals and the bra-ket referring to a functional integral on the space of all possible 3-geometries). Thus, in such a theory, we recognize of the evidence for a conserved probability amplitude $\Psi^{*} \Psi$ (being $\Psi^{*}$ the complex conjugate of the wave functional), with $\langle\Psi \mid \Psi\rangle=1$ and $\partial_{t}\langle\Psi \mid \Psi\rangle=0$.

Now we search for the link between the above scheme of quantization and the notion of reference fluid. To this end, we expand the wave functional as follows

$$
\Psi\left(t,\left\{h_{i j}\right\}, \phi\right)=\int_{* \mathcal{Y}_{t}} D \Omega \Theta(\Omega) \chi\left(\Omega,\left\{h_{i j}\right\}, \phi\right) \exp \left\{-\frac{i}{\hbar} \int_{t_{0}}^{t} d t^{\prime} \int_{\Sigma_{t}^{3}} d^{3} x(N \Omega)\right\}
$$


where $D \Omega$ denotes the Lebesgue measure in the functional space ${ }^{*} y_{t}$ of the conjugate function $\Omega\left(x^{i}\right)$ and $\Theta$ a functional valued in this domain. By this form of $\Psi$, equation (21) is reduced to the eigenvalues problem

$$
\left(\hat{H}^{g}+\hat{H}^{\phi}\right) \chi=\Omega \chi .
$$

Thus we see that, from a quantum point of view, the label time manifests its physical nature, via the appearance of a non-zero eigenvalue of the super-Hamiltonian.

In the limit $\hbar \rightarrow 0$, the WKB approximation, i.e. $\Psi \propto \exp \{i \sigma / \hbar\}$ provides an HamiltonJacobi operator which allows to identify the two quantities $\omega$ and $\Omega$, that is to say, on the classical limit, the energy density of the (dust) reference fluid is given by $\varepsilon=-\Omega / \sqrt{h}$; we will discuss in more detail below the nature of this identification (which is valid at all in general), with respect to the particular case of the closed FRW cosmology.

We see that, since in the coordinates system $\left\{t, x^{i}\right\}$, the fluid is "at rest" to the hypersurfaces $\Sigma_{t}^{3}$, then it contributes its energy density only to the super-Hamiltonian eigenvalue (in the limit $\hbar \rightarrow 0$ ); Therefore, even from our quantum analysis of the dynamics, it emerges

If the theory here proposed is a predictive one, we should expect to observe the trace of this reference fluid energy density from all those systems which underwent a classical limit; such a situation is surely true for our actual Universe and, indeed, we really observe (in the synchronous reference of our galaxy) an unidentified dust energy, the so-called dark matter; in the next two section, we will try to understand if it can exist a correlation between our dust fluid and the observed "matter component" of the Universe.

We observe that the restriction $\partial_{i} N=0$, required, on a classical point of view, for the validity of the dust fluid model, is not so relevant; in fact, the real physics is that one observed by the fluid reference its-self, (the synchronous one, in the coordinates $\left\{t, x^{i}\right\}$ ), as described by equations (23) (which generalize the Wheeler-Dewitt approach). In this sense, equation (21) provides only a different parameterization of the real physics.

To conclude, it is worth remarking how, the main difference between our approach and others interesting ones, that lead to the same formal issue (see the discussion in [1] about the comparison with the so-called "multi-time approach, as well as the formulations presented in [7, 8] and [12, 13]), consists of preliminary reducing the super-Hamiltonian to a linear form, and, overall, of setting ad hoc fields which play the role of time (for instance in 12, 13 is postulated,in the theory, the presence of a real mass-less scalar field). simply extend to the 3-metric dynamics the kinematical (embedding-like) action to provide physical meaning in the splitting procedure, and then interpret it as a dust fluid (with the role of time). In this scheme the 3-metric is related to the space-time one by the dynamical field $y^{\mu}$, so, heuristically, we can say to bypass the theory background independence.

\section{The Closed FRW Cosmology}

Since the clock by which we are measuring the age of the Universe is (essentially) a synchronous one, and we expect the cosmological dynamics became a classical one, then 
the contribution of the "dust fluid" energy density must appear in the galaxies recession. Below we will face the questions about the modifications introduced, by our approach, in the quantum evolution of the Universe, and about the actual value of the dust energy density.

We investigate the quantum dynamics predicted, in a synchronous reference, by equation (21) for the closed Friedmann-Robertson-Walker model [14, 15, whose line element reads (below we adopt the standard notations for the fundamental constants)

$d s^{2}=-c^{2} d t^{2}+R_{c}^{2}(t)\left[d \xi^{2}+\sin ^{2} \xi\left(d \eta^{2}+\sin ^{2} \eta d \phi^{2}\right)\right] \quad 0 \leq \xi<\pi 0 \leq \eta<\pi 0 \leq \phi<2 \pi$.

Here $R_{c}$ denotes the radius of curvature of the Universe, measurable, in principle, via the relation $R_{c}=c /(H \sqrt{\Omega-1})$ (being $H$ the Hubble function, $\bar{\Omega}$ the critical parameter and $\left.R_{c(\text { today })} \sim \mathcal{O}\left(10^{28} \mathrm{~cm}\right)\right)$.

In the very early phases of the Universe evolution, it is expected a space filled by a thermal bath, involving all the fundamental particles; since, at very high temperatures, all the massive particles are ultrarelativistic ones, then the most appropriate phenomenological representation of the matter-radiation thermal bath, is provided by an energy density of the form $\mu^{2} / R_{c}^{4}$.

Furthermore, the idea that the Universe underwent an inflationary scenario, leads us to include $a b$ initio in the dynamics a real self-interacting scalar field $\phi$, described by a "finite-temperature" potential $V_{T}(\phi)$ (here $T$ denotes the thermal bath temperature), which we may take, for instance, in the Coleman-Weinberg form

$V_{T}(\phi)=\frac{B \sigma^{4}}{2 h^{3} c^{3}}+B \frac{\phi^{4}}{h c}\left[\ln \left(\frac{l_{P l} \phi^{2}}{\sigma^{2}}\right)-\frac{1}{2}\right]+\frac{1}{2} m_{T}^{2} \phi^{2} \quad m_{T}=\sqrt{\lambda T^{2}-m^{2}} \quad(m, \lambda)=$ const.

where $B$ is a parameter related to the fundamental constraints of the theory (estimated $\mathcal{O}\left(10^{-3}\right), \sigma$ corresponds to the energy scale associated with the symmetry breaking process (i.e. $\left.\sigma \sim \mathcal{O}\left(10^{15}\right) G e V\right)$ ), while $m$ and $l_{P l}$ denote, respectively, the inverse of a characteristic lenght and $l_{P l}$ the Planck length $l_{P l} \equiv \sqrt{G \hbar / c^{3}}$.; the temperature dependence of the potential term can be also regarded as a time evolution of the model. The dynamics of such a cosmological model is summarized, as shown when developing the Einstein-Hilbert action under the present symmetries, by the Hamiltonian function

$$
\frac{\mathcal{H}}{c}=-\frac{l_{P l}^{2}}{3 \pi \hbar} \frac{p_{R_{c}}^{2}}{R_{c}}+\frac{c}{4 \pi^{2}} \frac{p_{\phi}^{2}}{R_{c}^{3}}+\frac{\mu^{2}}{R_{c}}-\frac{3 \pi \hbar}{4 l_{P l}^{2}} R_{c}+2 \pi^{2} R_{c}^{3} V_{T}(\phi),
$$

with $p_{R_{c}}$ and $p_{\phi}$ being the conjugate momenta to $R_{c}$ and $\phi$.

Thus, the Schrödinger equation (21) reads, once turned the above Hamiltonian into an operator (which possesses the right normal ordering), as follows 


$$
\frac{i \hbar}{c} \partial_{t} \Psi\left(t, R_{c}, \phi\right)=\left\{\frac{l_{P l}^{2} \hbar}{3 \pi} \partial_{R_{c}} \frac{1}{R_{c}} \partial_{R_{c}}-\frac{\hbar^{2} c}{4 \pi^{2}} \frac{1}{R_{c}^{3}} \partial_{\phi}^{2}+\frac{\mu^{2}}{R_{c}}-\frac{3 \pi \hbar}{4 l_{P l}^{2}} R_{c}+2 \pi^{2} R_{c}^{3} V_{T}(\phi)\right\} \Psi\left(t, R_{c}, \phi\right),
$$

Before going on with the analysis of this equation, we need to precise some aspects concerning the potential term relevance during the Universe evolution.

It is well-known that the classical scalar field dynamics is governed by the following equation

$$
\ddot{\phi}+3 H \dot{\phi}+c^{2} \hbar^{2} \frac{d V_{T}}{d \phi}=0 .
$$

The presence of the potential term is surely crucial to generate the inflationary scenario, but, sufficiently close to the initial "Big-Bang", its dynamical role is expected to be very limited; in fact, if we neglect the potential term in (28), then, remembering that for early times $R_{c} \sim \sqrt{t} \rightarrow H \sim 1 / 2 t$, we get the free field solution $\phi \propto \ln t$. Now the terms we retained to solve equation (28) are potentially of the order $\mathcal{O}\left(1 / t^{2}\right)$; in the limit toward the "Big-Bang" $(t \rightarrow 0)$, the potential term (25) (we recall that $\left.T \propto 1 / R_{c} \propto{ }^{\prime} 1 / \sqrt{t}\right)$ can be clearly negligible, i.e. $t^{2} V_{T(t)}(\phi(t)) \rightarrow 0$. Apart from very peculiar stiff cases, all the inflationary potentials result to be negligible at very high temperatures.

Taking into account the above classical analysis, we may assume that, during the Planck epoch, when the Universe performed its quantum evolution, the potential of the scalar field plies no significant role; therefore, by choosing the following expansion for the wave function

$$
\Psi\left(t, R_{c}, \phi\right)=\int_{-\infty}^{\infty} \int_{-\infty}^{\infty} d \epsilon d p C(\epsilon, p) \theta\left(\epsilon, p R_{c}\right) \exp \left\{\frac{i}{\hbar}(p \phi-\epsilon t)\right\},
$$

(with $C(\epsilon, p)$ denoting generic coefficients), we get, from (27), the eigenvalues problem

$$
\left\{\frac{l_{P l}^{2} \hbar}{3 \pi} \frac{d}{d R_{c}} \frac{1}{R_{c}} \frac{d}{d R_{c}}+\frac{p^{2} c}{4 \pi^{2}} \frac{1}{R_{c}^{3}}+\frac{\mu^{2}}{R_{c}}-\frac{3 \pi \hbar}{4 l_{P l}^{2}} R_{c}\right\} \theta=\frac{\epsilon}{c} \theta .
$$

with the boundary conditions $\theta\left(R_{c}=0\right)=0$ and $\theta\left(R_{c} \rightarrow \infty\right)=0$.

A solution to this equation reads in the form

$$
\theta \propto \sqrt{R_{c}} \exp \left\{-\frac{\left(R_{c}-R_{c(0)}\right)^{2}}{4 \alpha^{2}}\right\} ;
$$

in order to be the above functional form a solution of equation (30), we have to require the relations $p= \pm \sqrt{\pi \hbar / c} l_{P l}, \alpha=l_{P l} / \sqrt{3 \pi}$ and $\epsilon=-3 \pi \hbar c R_{c(0)} / 2 l_{P l}^{2}$. Furthermore, since the ultrarelativistic energy density is manifestly positive, then, from the following expression for $\mu^{2}$

$$
\mu^{2}=\frac{l_{P l}^{2}}{3 \pi}\left(\frac{1}{2 \alpha^{2}}-\frac{R_{c(0)}^{2}}{4 \alpha^{4}}\right)
$$


we find an important restriction on the continuous eigenvalue spectrum, i.e. $-\sqrt{3 \pi / 2} M_{P l} c^{2}<$ $\epsilon<\sqrt{3 \pi / 2} M_{p l} c^{2}$ (being $M_{p l}$ the Planck mass, $M_{p l}=\hbar / c l_{P l}$ ).

Thus, we get a (non-normalizable) probability amplitude, for the stationary states, of the form

$$
P_{\text {Stat }} \propto \cos ^{2}(|p| \phi) R_{c} \exp \left\{-\frac{\left(R_{c}-R_{c(0)}\right)^{2}}{2 \alpha^{2}}\right\} .
$$

The $\phi$-component of the wave function is not normalizable, because of the potential field absence (we have to do with a situation analogous to that one of a free nonrelativistic particle admitting only two momentum eigenvalues), but it is remarkable the existence, as effect of our revised quantization approach, of stationary states for the radius of curvature; in the obtained dynamics, we see that the notion of the cosmological singularity is replaced by the more physical one of a peaked probability to find $R_{c}$ near zero. The approximation of neglecting the potential term $V_{T}$ can be regarded as confirmed a posteriori by the small probability that the system penetrates regions where $R_{c}$ is much greater than the Planck length and the temperature is sufficiently small to be compared with the symmetry breaking scale.

In order to construct the semiclassical limit of equation (30), we separate $\theta$ into its modulus and phase, i.e. $\theta=\sqrt{\alpha} \exp \{i \beta / \hbar\}$; then we get the following two, real and complex, components of equation (30)

$$
\begin{gathered}
-\frac{l_{P l}^{2}}{3 \pi \hbar} \frac{1}{R_{c}}\left(\frac{d \beta}{d R_{c}}\right)^{2}+\frac{p^{2} c}{4 \pi^{2}} \frac{1}{R_{c}^{3}}+\frac{\mu^{2}}{R_{c}}-\frac{3 \pi \hbar}{4 l_{P l}^{2}} R_{c}-\frac{\epsilon}{c}+\hbar^{2} V_{\text {Quantum }}=0 \\
\frac{1}{\sqrt{\alpha}} \frac{d}{d R_{c}}\left(\frac{\alpha}{R_{c}} \frac{d \beta}{d R_{c}}\right)=0 \Rightarrow \alpha \propto R_{c} /\left(d \beta / d R_{c}\right), \\
V_{\text {Quantum }} \propto \frac{1}{\sqrt{\alpha}} \frac{d}{d R_{c}}\left(\frac{1}{R_{c}} \frac{d \sqrt{\alpha}}{d R_{c}}\right) .
\end{gathered}
$$

In the limit $\hbar \rightarrow 0$, when becomes negligible $V_{\text {Quantum }}$, we reobtain the HamiltonJacobi equation describing the Universe classical dynamics, but with an additional term corresponding to a non-relativistic matter contribution, which, when $\epsilon$ is negative, acquires positive energy density; to this respect, we remark how, on the quantum level, the Universe is expected to approach the lowest, i.e. negative, energy state.

We stress how, for sufficiently large $R_{c}$, if the non-relativistic term dominates (the spatial curvature being yet negligible), then we get $d \beta / d R_{c} \propto \sqrt{R_{c}}$ and therefore $R_{c} \rightarrow \infty \Rightarrow V_{\text {Quantum }} \sim 1 /\left(R_{c}^{3}\right) \rightarrow 0$; such a behavior supports the idea that, when the Universe "expands enough" (i. e. its volume fluctuating explores regions of high $R_{c}$ values), it can approach a classical dynamics.

The analysis of this section answers the question about the cosmological phenomenology implied by our approach and the issue goes toward the appearance, in a synchronous reference, of a pressureless contribution to the Universe energy density. In the next section, we make some estimations in order to understand if such a new term (which is 
nothing more than the classical limit of the total Universe quantum energy) may have something to do with the observed dark matter component.

\section{Phenomenological Considerations}

Indeed, by adding a term to the gravitational action, we may expect it appears as a new kind of energy-momentum term; what makes our analysis a valuable one is in the following points:

i) The kinematical action is an embedding-like geometrical object, whose existence in quantum gravity, was postulated in [1] on the base of well-grounded statements and not invented ad hoc. Above we have shown that it can be interpreted, from a classical point of view, as a non-relativistic dust fluid; a non-relativistic energy density is also what appears from the quantum dynamics, when taking the classical limit.

ii) All the accepted models of cold dark matter predict the existence of a very early (decoupled) zero-pressure component, able, by this feature, to develop large scale structures (at the present time even the heat dark matter is expected to be non-relativistic). Indeed, a non-baryonic component of this kind, is estimated (either by the supernova data, either by the cosmic microwaves background (detected) anisotropy) to be $\sim 0.3$ of the actual Universe critical density.

Since in equation (34) $\beta$ plies the role of the (reduced) action function, we can write, by using Hamilton equations, the following relation ${ }^{2}$

$$
\frac{d \beta}{d R_{c}}=p_{R_{c}}=-\frac{3 \pi \hbar}{2 c l_{P l}^{2}} R_{c} \frac{d R_{c}}{d t} .
$$

Then, remembering that $H=\left(d R_{c} / d t\right) / R_{c}$ and $\bar{\Omega}-1=c^{2} / H^{2} R_{c}^{2}$, we see how equation (34) takes the simple form (with obvious notation for the different contributions) $\sum_{i} X_{i}=$ 1, being $X_{i} \equiv \bar{\Omega}_{i} / \bar{\Omega}(i=p, \mu, d m$, curv $)$; thus, our dust fluid provides a component of the critical parameter $\bar{\Omega}_{d m}$, given by

$$
\bar{\Omega}_{d m}=-\frac{4 l_{P l}^{2} c \epsilon}{3 \pi \hbar H^{2} R_{c}^{3}} .
$$

Such a formula is valid in general, independently of the other kinds of matter present in the universe, and, therefore, provides a good tool to investigate the role it could play in the actual cosmology; in this respect, we stress the following three relevant points:

i) If we take for $\epsilon$ the minimum value of the continuous spectrum obtained in the previous section, within the framework of a "pre-inflationary" scenario, i.e. $\epsilon \sim \mathcal{O}\left(-M_{P l} c^{2}\right)$, then we get

\footnotetext{
${ }^{2}$ the same result could be directly obtained by applying the Hamilton-Jacobi method to the full action $\mathcal{S}=\beta\left(R_{c}\right)+p \phi-\epsilon t$.
} 


$$
\bar{\Omega}_{d m}=\mathcal{O}\left(\frac{l_{P l} c^{2} H^{-2}}{R_{c}^{3}}\right) \sim \mathcal{O}\left(10^{-63}\right) .
$$

ii) The value of $\epsilon$, required to have $\bar{\Omega}_{d m}=\mathcal{O}(1)$ (so that it could make account for the real dark matter component, estimated about 0.3 of the actual critical density), corresponds to

$$
\epsilon^{*} \sim \mathcal{O}\left(\frac{\hbar c R_{c}^{3}}{l_{P l}^{2} c^{2} H^{-2}}\right) \sim \mathcal{O}\left(10^{82} G e V\right) ;
$$

such a value corresponds to the present one of the total energy of the Universe, whether it admits a closed space. A crucial point is that $\epsilon$ is a constant of the motion and therefore, since the Universe became a classical one, it was characterized by such value $\epsilon^{*}$.

iii) In order to get an inflationary scenario, able to explain the paradoxes of the Standard Cosmological Model, we need a sufficiently large "e-folding" which allows the size of an horizon, at the inflation beginning, be now of the order of the actual Hubble radius; such a value corresponds, at least, to about 60 , i.e. the ratio between the scale factors, respectively, after and before the inflation, is around a factor $\mathcal{O}\left(10^{26}\right)$. This means that, if today $R_{c} \sim \mathcal{O}\left(10^{28} \mathrm{~cm}\right)$, then, taking into account that the redshift of the end of the inflation is about $z \sim \mathcal{O}\left(10^{24}\right)$, we see that when the de-Sitter phase started its value was $R_{c} \sim \mathcal{O}\left(10^{-22} \mathrm{~cm}\right)$. Thus, the total energy of the Universe, when the dynamics became dominated by the "vacuum energy" at the temperature $\sigma \sim \mathcal{O}\left(10^{15} \mathrm{GeV}\right)$, is given by the expression

$$
\epsilon_{\Lambda} \sim \frac{\sigma^{4} R_{c}^{3}}{h^{3} c^{3}} \sim \mathcal{O}\left(10^{36} G e V\right) \ll \epsilon^{*} ;
$$

this result seems to indicate that, assuming the Universe underwent an inflationary scenario, we get the contradictory issue about the impossibility of a dominating "vacuum energy".

Summarizing, the above considerations are against the idea that the here obtained $\bar{\Omega}_{d m}$ can make account for the dark matter, if inflation took place. The situation is different if we take the picture of the Standard Cosmological Model because, for instance, a classical estimation of the thermal bath energy at the Planck epoch is about $\mathcal{O}\left(\left(R_{c} / l_{P l}\right)^{3} M_{P l} c^{2}\right) \sim$ $\mathcal{O}\left(10^{112} \mathrm{GeV}\right)$; thus, in absence of inflation, the value of $\epsilon^{*}$ would have become important only in the later stage of the Universe evolution and it could play today a relevant dynamical role.

\section{References}

[1] G. Montani, Nucl. Phys. B, (2002), 634, 370. 
[2] K. Kuchar, in Quantum Gravity II, a second Oxford symposium, (1981), eds C. J. Isham et al., Clarendom Press., Oxford,

[3] R. Arnowitt, S. Deser and C, W. Misner, in Gravitation: an introduction to current research, (1962), eds I. Witten and J. Wiley, New York.

[4] B. S. DeWitt, Phys. Rev., (1967), 160, 1113.

[5] B. S. DeWitt, Proc. eighth Marcell Grossmann meeting, (Jerusalem, 22-27 June 1997), ed T. Piran, 6.

[6] C. J. Isham, Canonical Quantum Gravity and the Problem of Time, (1992), available /arxiv:/gr-qc/9201011.

[7] C. Rovelli, (1991). Class. and Quantum. Grav. , 8 (1991) 297.

[8] C. Rovelli, (1991), Phys. Rev. D, 43, 442.

[9] K. Kuchar and C. Torre, Phys. Rev. D, (1991), 43, 419.

[10] J. D. Brown and K. V. Kuchar, Phys. Rev. D, 51, (1995), 5600.

[11] J. Bick and K. V. Kuchar, Phys. Rev. D, 56, (1997), 4878.

[12] L. Smolin, (1993). available arxiv:gr-qc/9301016.

[13] C. Rovelli and L Smolin, (1993), available arxiv:gr-qc/9308002.

[14] E. W. Kolb and M. S. Turner, The Early Universe, (1990), (Adison-Wesley, Reading), 447.

[15] J. B. Hartle, in Highlights in Gravitation and Cosmology (1988), eds B. Iver et al., Cambridge Univ. Press. 